\title{
A PHENOMENOLOGICAL IMAGINARY PART OF THE OPTICAL POTENTIAL FOR HEAVY IONS
}

\author{
J.M. QUESADA, M. LOZANO and G. MADURGA \\ Departamento de Física Atómica y Nuclear, Facultad de Física, Universidad de Sevilla, Apdo. 1065, Sevilla, Spain
}

Received 19 January 1983

Revised manuscript received 2 March 1983

\begin{abstract}
The depth of the inaginary part of the oplical potential is derived from the assumption that, at a given energy and for each partial wave $\mathrm{L}$, it is proportional to the compound nucleus density level up to a given excitation energy above the yrast level corresponding to the angular momentum $L$, and remains a constant for smaller values of $L$. The prescription is successfully tested for the system ${ }^{16} \mathrm{O}+{ }^{28} \mathrm{Si}$ at nine different projectile energies between 33 and $81 \mathrm{MeV}$; it fails however at $141.5 \mathrm{MeV}$, as expected, because other channels, besides elastic scattering and fusion, are important.
\end{abstract}

In heavy-ion physics the large decrease of the elastic cross section at energies where the nuclei begin to penetrate each other points to a large absorption out of the elastic channel. This is usually described by means of the imaginary potential in the optical model. During the last decade much effort has been made to derive this potential from different approaches [1-4]. In this paper, following a suggestion by Arima and Hodgson [5], we present a method to construct the inaginary part of the optical potential. This is inspired, in part, in the model proposed by Helling et al. [6] where that potential is given by the transition probability from the elastic channel into a precompound state which is a doorway state for the formation of the compound nucleus. This transition probability is given in first order by Fermi's golden rule:

$\mathrm{d} w / \mathrm{d} t=(2 \pi / \hbar)\left|\left\langle\psi_{\text {comp }}\left|V_{\text {int }}\right| \psi_{\text {elas }}\right\rangle\right|^{2} \rho\left(E^{*}, L\right)$,

where $\rho\left(E^{*}, L\right)$ is the level density of the precompound nucleus with excitation energy $E^{*}$ and angular momentum $L . V_{\text {int }}$ is the interaction between the precompound state $\psi_{\text {comp }}$ and the elastic channel $\psi_{\text {elas }}$. To estimate the magnitude of the transition matrix element it is necessary to introduce microscopic wave functions. Because the nucleons in the overlap region contribute most to the transition probability, Fink et al. [7] assume that the radial dependence of the square of the matrix element in (1) is proportional to the number of nucleons in the overlap region. This suggests a factorization of the imaginary potential:

$W(r, E, L)=W(r) W(E, L)$,

where $W(r)$ contains the radial dependence and $W(E, L)$ the energy and angular momentum dependence through the level density $\rho\left(E^{*}, L\right)$ of the compound nucleus. This approximation implies that the formation of the compound nucleus is the dominant reaction mechanism. Such an assumption is reasonable only for not too high energy and not very heavy ions. This is one first limitation of our model.

The yrast level is the state of highest possible angular momentum for a given excitation energy. Since angular rotations have usually the highest angular momentum, for a given energy the yrast level is reached if all excitation energy is converted into rotational energy. Bellow the yrast line of the compound nucleus the imaginary potential (2) is exactly zero since no compound states can be reached. Based on these ideas the imaginary part (2) of the optical potential may be expressed as follows:

$$
\begin{aligned}
& W(r)=\{1+\exp [(r-R) / a]\}^{-1}, \\
& R=r_{0}\left(A_{1}^{1 / 3}+A_{2}^{1 / 3}\right),
\end{aligned}
$$


$W(E, L)=0, \quad$ for $E^{*}<E_{\mathrm{y}}(L)$

$W(E, L)=c \rho\left(E^{*}, L\right), \quad$ for $E_{\mathrm{y}}(L)<E^{*}<E_{\mathrm{y}}(L)+E_{1}$,

$W(E, L)=c_{0}+c_{1} E, \quad$ for $E^{*}>E_{\mathrm{y}}(L)+E_{1},(3$ cont'd $)$

where $E_{\mathrm{y}}(L)$ is the yrast energy for compound nucleus states with spin $L$, and $E$ is the center of mass kinetic energy of the entrance channel. Above a limit energy $E_{1}$ the level density becomes so high that the probabilities of exciting different states compete with each other and the total probability is no longer a sum of independent probabilities. The model simplifies this effect by assigning a saturation value $W(E, L)$, linear in $E$, for energies above a limit $E_{\mathrm{y}}+E_{1}$. In our model $E_{1}, c_{0}$ and $c_{1}$ are the model constants to be determined and $c$ is fixed by continuity.

Expression (3) implies the assumption that the formation of the compound nucleus is determined by the yrast energy. It has been shown [8], however, that in some reactions, specially for $2 \mathrm{~s}-1 \mathrm{~d}$ nuclei, the fusion is limited by dynamical processes in the entrance channel rather than by intrinsic characteristics of the compound nucleus. If this is to be generalized, the experimental limit $E^{*}\left(L_{\mathrm{c}}\right)$ should be introduced in (3) instead of the yrast limit (here $L_{\mathrm{c}}$ is the critical angular momentum for the compound nucleus formation which is dependent on the $E^{*}$ ). When the experimental relation $E^{*}$ versus $L_{\mathrm{c}}$ is not available, the semiempirical model of Glas and Mosel [9] can provide an approximation for it. Nevertheless, we have preferred to be more systematic, for the sake of simplicity, and hope that the different approaches can be compensated by an adequate choice of the parameters, particularly $E_{1}$.

The essential ingredient in the model is the level density. There are many statistical formulae for $\rho\left(E^{*}, L\right)$ but we choose that of Kataria et al. [10] for three reasons: first it takes into account the influence of the nuclear shell structure on the level density and the excitation energy dependence of shell effects; secondly it is capable to take into account the nuclear deformation; and finally it gives an acceptable fit to the experimental data on neutron resonance spacings and it also provides a reliable extrapolation to higher excitation energies.

It is known that the level density is related to the state density $W\left(E^{*}\right)$ by the expression:

$$
\begin{aligned}
& \rho\left(E^{*}, L\right)=\left[(2 L+1) W\left(E^{*}\right) / 2(2 \pi)^{1 / 2} \sigma^{3}\left(E^{*}\right)\right] \\
& \quad \times \exp \left[-L(L+1) / 2 \sigma^{2}\left(E^{*}\right)\right]
\end{aligned}
$$

where the spin cut-off factor $\sigma^{2}$ is related to the moment of inertia of the nucleus by the relation:

$\sigma^{2}=J T / \hbar^{2}$,

$T$ being the temperature. The state density is mainly determined by the entropy $S\left(E^{*}\right)$ of the nucleus by the following expression:

$W\left(E^{*}\right)=\exp \left[S\left(E^{*}\right)\right] /(2 \pi)^{3 / 2} D^{1 / 2}=C \exp \left[S\left(E^{*}\right)\right]$.

The constants $D$ and $C$ are determined by the following well-known relations:

$D=\left(\pi^{2} / 12\right) g_{0}^{3} T^{5}, \quad C=\sqrt{\pi /}\left(12 a^{1 / 4} E^{* 5 / 4}\right)$,

where $g_{0}$ is the density of the single-particle states, related to the Liquid Drop Model (LDM) value of the level density parameter $a$ as:

$a=\frac{1}{6} \pi^{2} g_{0}$.

The analytical expression for the entropy as a function of the temperature, obtained by Gilbert [11], and the corresponding expression for the excitation energy are approximated by Kataria et al. [10] in such a way that they can be written as:

$$
\begin{aligned}
S= & 2 a T+\left(\Delta_{\mathrm{S}} / T\right)\left[\pi^{2} \omega^{2} T^{2} \cosh (\pi \omega T) / \sinh ^{2}(\pi \omega T)\right. \\
& -\pi \omega T / \sinh (\pi \omega T)],
\end{aligned}
$$

and

$E^{*}=a T^{2}+\Delta_{S}\left[\pi^{2} \omega^{2} T^{2} \cosh (\pi \omega T) / \sinh ^{2}(\pi \omega T)-1\right]$,

where $\Delta_{\mathrm{s}}$ is the ground state shell correction energy, i.e., the difference in the ground state energies between the actual nucleus and the corresponding smooth system. The values for $\Delta_{\mathrm{s}}$ were obtained as the differences between the experimental binding energy and the LDM energy obtained with parameters of Seeger and Howard [12]. The constant $\omega$ is related to the major shell spacing $\lambda$ simply through $\omega=2 \pi / \lambda$. It is known that $\lambda=k / A^{1 / 3}$ where $k$ is expected to be around 30 to $40 \mathrm{MeV}$. Hence one can write $\omega$ $=\omega_{0} A^{1 / 3}$ where $\omega_{0}=2 \pi / k$ is a mass independent parameter with a value [10] equal to $0.185 \mathrm{MeV}^{-1}$. The 
effect of nucleon pairing is accounted for by substitution of an effective excitation energy for the true excitation energy: $E^{* \prime}=E^{*}-\Delta_{\mathrm{p}}$, where $\Delta_{\mathrm{p}}$ is equal to the pairing energy values of Gilbert and Cameron [13].

For a noninteracting Fermi gas confined to a finite volume the level parameter can be expressed as:

$a=\gamma A\left(1-\beta B_{\mathrm{s}} A^{-1 / 3}\right)$,

where $\gamma=0.176 \mathrm{MeV}^{-1}$ and $\beta=1.0 . B_{\mathrm{s}}$ is the usual nuclear surface area relative to spherical shape. At excitation energies below $10 \mathrm{MeV}$ the nuclear deformation coincides with that of the ground state for which $B_{\mathrm{s}}$ is well known. This is not our case. For higher excitation energies we calculate $B_{\mathrm{s}}$ from the approximation [14] for the moment of inertia obtained in the LDM framework:

$J_{\text {nucl }}=J_{\text {rig }}\left(1+\delta L^{2}\right)$,

where $\delta$ is a constant specific for each nucleus. Assuming conservation of the nuclear volume and that for the range of angular momentum of interest the nucleus adopts an oblate ellipsoidal shape we have found:

$$
\begin{gathered}
B_{s}(L)=\frac{1}{2}\left\{\mu+\left[\mu\left(\mu^{3}-1\right)\right]^{-1 / 2}\right. \\
\left.\quad \times \ln \left[\mu^{3 / 2}+\left(\mu^{3}-1\right)^{1 / 2}\right]\right\},
\end{gathered}
$$

with $\mu=1+\delta L^{2}$. Fitting to LDM results for a number of nuclei, we found the relation:

$\delta=0.23 A^{-1.56}$.

As an application of the model we have studied the reaction ${ }^{16} \mathrm{O}+{ }^{28} \mathrm{Si}$ at 10 different projectile energies: $E_{\text {lab }}=33,36,38,50,53,55,66,72,81$, and 141.5 $\mathrm{MeV}$. The constants in (3) were determined by fitting the elastic experimental data [15]. To do it we have used the Niels Bohr Institute version of the GENOA code [16] modified by introducing our $L$-dependent imaginary potential. For the real part we have used a folding potential obtained with a density-dependent effective interaction [17]. The criterion to determine the constants of the model was the minimization of the $\chi^{2}$ defined as usual. Two preliminary points were apparent in the first calculations. The fitted value of $E_{1}$ is around $10 \mathrm{MeV}$ which turns out to switch off the $\rho\left(E^{*}, L\right)$ proportionality when the slope of $\rho$ versus $L$ becomes significantly steeper. On the other hand, the scattering data at $141.5 \mathrm{MeV}$ did not admit a reasonable fit within the model. This fact is consistent

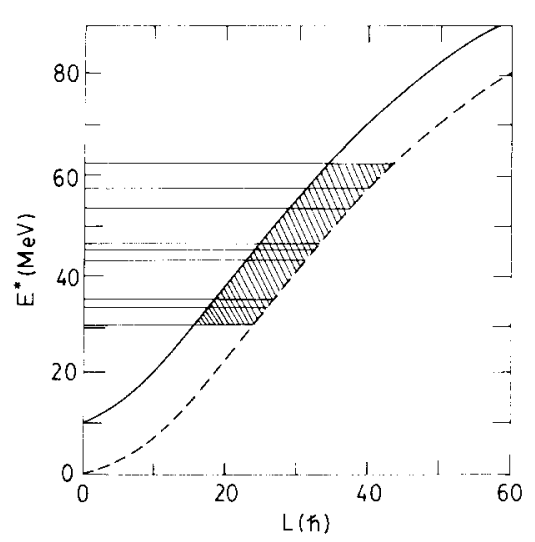

Fig. 1. Yrast line (dashed) and yrast $+E_{1}$ (solid line) with the $\rho$-proportionality zone shaded. Horizontal lines correspond to the compound nucleus excitation energy in the nine sets of data fitted.

with the basic assumption that the compound nucleus formation must be the dominant reaction mechanism: at that energy the competition of other reaction channels is so important that the model is not adequate at all.

Fig. 1 shows the area of level density proportionality of $W(E, L)$ for ${ }^{44} \mathrm{Ti}$, in an $\left(E^{*}, L\right)$ plane, limited by $E_{\mathrm{y}}(L)$ and $E_{\mathrm{y}}+10$. The yrast line has been determined with the moment of inertia defined by eqs. (12) and (14). Since the imaginary potential is proportional to the density of states, for a given $E$ the absorption will also be largest from the upper line to the left (smaller $L$ ) due to the nature of the $E_{1}$ limit. This is strictly accurate only if the transition matrix elements in (1) are independent of $E$ and $L$, as expressed by eq. (2). The level density and hence $W(E, L)$ are rapidly decreasing from the upper line towards the yrast curve (larger $L$ ).

The value of the constants $c_{0}$ and $c_{1}$ in (3) obtained by fitting simultaneously the 9 experimental angular distributions can be established as $c_{0}=26$ $\mathrm{MeV}$ and $c_{1}=-0.2$. In fig. 2 the elastic scattering angular distributions predicted by the model are compared to the experimental data used in the fit. The total reaction cross sections deduced from the resulting optical potentials are compared to the experimental fusion cross sections of ref. [18] in fig. 3. The theoretical reaction cross sections are about $15 \%$ above the experimental data. This small inconsistency with the underlying idea of fusion being the only reaction channel, may derive from the use of the yrast limit 


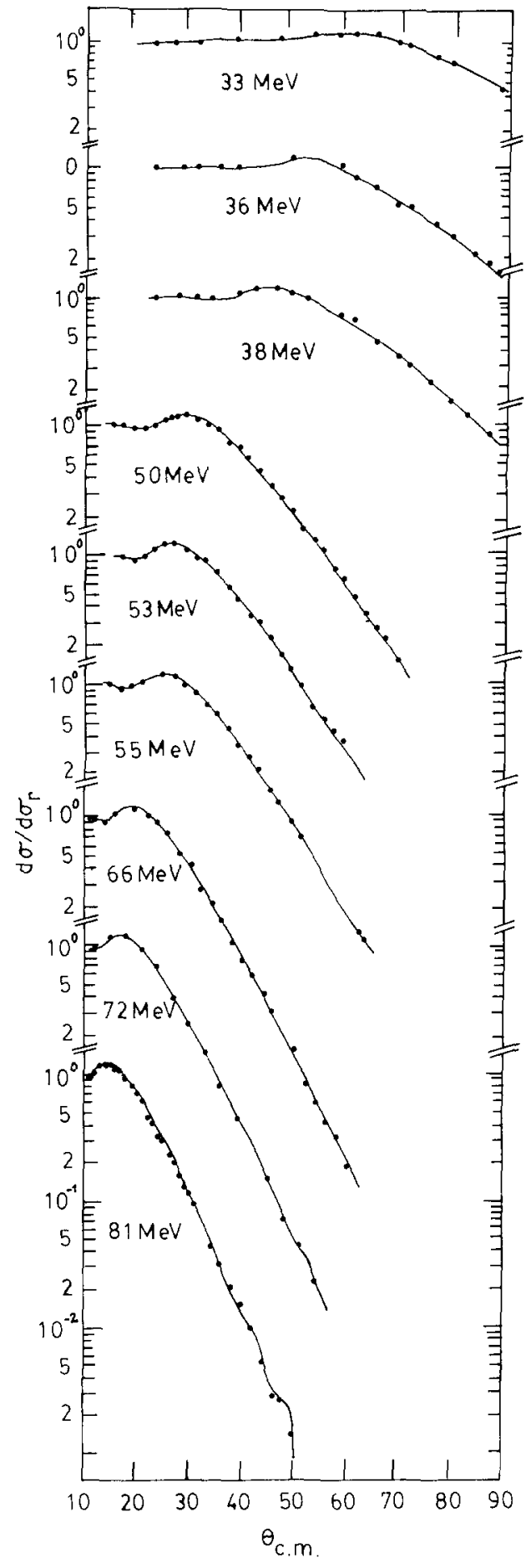

Fig. 2. Elastic scattering angular distributions predicted in this work for the system ${ }^{16} \mathrm{O}+{ }^{28} \mathrm{Si}$ are compared to experimental data at 9 different energies between 33 and $81 \mathrm{MeV}$.

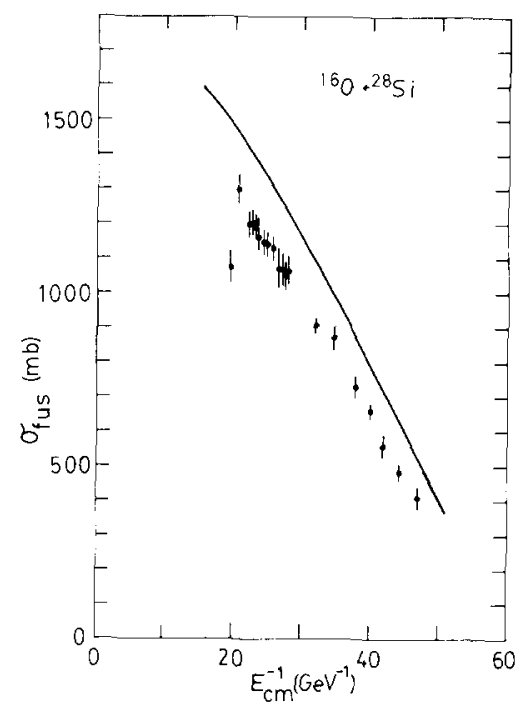

Fig. 3. Data on the fusion cross section of ${ }^{16} \mathrm{O}+{ }^{28} \mathrm{Si}$ from Rascher et al. [18] are compared with the total reaction cross sections deduced from the resulting optical potentials.

rather than the experimental one, as mentioned above.

We have also checked that the exact value of $E_{1}$ can be modified within a wide range, from 7 to 12 $\mathrm{MeV}$, provided that the constants are adjusted within certain limits, $c_{0}=26.0 \pm 0.8 \mathrm{MeV}$ and $c_{1}=-0.20$ \pm 0.02 , and the quality of the fit does not sensibly change. With smaller or higher values of $E_{1}$ the quality of the fit at the same energies of our range worsens so that a simultaneous fit to all of the data is not possible.

In summary we see that the idea of relating the $L$ dependent depth of the imaginary potential to the compound nucleus level density looks very useful for the fitting of elastic scattering data and deserves further attention. The data thus far analyzed are smooth and restricted to forward angles. The success of the model encourages us to apply it in a further work to systems with a wider range of angles and showing some structure.

The authors are grateful to P.E. Hodgson and A. Arima for useful discussions, and to the Comision Asesora de Investigación Científica y Técnica for finan. cial support.

\section{References}

[1] A. Gobbi et al., Phys. Rev. C7 (1973) 30. 
[2] W. Greiner, in: Proc. Intern. Conf. on Dynamical properties of heavy-ion reactions (Johannesburg, South Africa, 1978), South African J. Phys. 1 (1978) 75.

[3] T. Izumoto, S. Krewald and A. Faessler, Nucl. Phys. A357 (1981) 471.

[4] R.A. Broglia, G. Pollarolo and A. Winther, Nucl. Phys. A361 (1981) 307.

[5] A. Arima and P.E. Hodgson, private communication.

[6] G. Helling, W. Scheid and W. Greiner, Phys. Lett. 36B (1971) 64 .

[7] H.J. Fink, W. Scheid and W. Greiner, Nucl. Phys. A188 (1972) 259.

[8] S. Harar, Proc. Symp. on Deep-inelastic and fusion reactions with heavy ions (Berlin, 1979), ed. W. von Oertzen, Lecture Notes in Physics, Vol. 117 (Springer, Berlin) p. 367

[9] D. Glas and U. Mosel, Phys. Lett. 78B (1978) 9.
[10] S.K. Kataria, V.S. Ramamurthy and S.S. Kapoor, Phys. Rev. C1 8 (1980) 549.

[11] A. Gilbert, University of California Radiation Laboratory Report No. UCRL'18095 (1968), unpublished.

[12] P.A. Seeger and W.M. Howard, Nucl. Phys. A238 (1975) 491.

[13] A. Gilbert and A.G.W. Cameron, Can. J. Phys. 43 (1965) 1446.

[14] F. Pühlhofer, Nucl. Phys. A280 (1977) 267.

[15] J.G. Cramer et al., Phys. Rev. C14 (1976) 2158.

[16] F.G. Perey, unpublished; B. Nilsson, private communication.

[17] F.J. Viñas, M. Lozano and G. Madurga, Phys. Rev. C23 (1981) 780 .

[18] R. Rascher, W.F.J. Müller and K.P. Lieb, Phys. Rev. C20 (1979) 1028 . 